\title{
Orthogonal Poly-Phase MC-CDMA over Multipath Power-Line Channels with Middleton Class-A Noise
}

\author{
Khaled M. Rabie and Emad Alsusa \\ School of Electrical and Electronic Engineering, \\ The University of Manchester, Manchester, UK, M13 9PL, \\ Email: \{khaled.rabie, e.alsusa $@$ manchester.ac.uk
}

\begin{abstract}
This paper investigates the application of orthogonal poly-phase based multi-carrier code division multiple access (OPP-MC-CDMA) over multipath power-line channels perturbed by Middleton class-A noise. The proposed OPP-MC-CDMA system is aided with a minimum-mean square error equalizer combined with nonlinear preprocessing to overcome the effect of bursty noise and multipath fading. We study the performance of this system in terms of the output signal-to-noise ratio (SNR) and symbol error rate with various constellation sizes of OPP codes, different noise scenarios and non-linear processor's thresholds. For comparisonsake, the performance of a conventional orthogonal frequency-division multiple access (OFDM) scheme is included. The results reveal that the proposed approach always provides superior performance over the conventional OFDM system with a maximum output SNR gain of up to $5.25 \mathrm{~dB}$. It is also shown that the performance of the OPP-MC-CDMA technique improves significantly with increasing the constellation size of the OPP codes.
\end{abstract}

Index Terms-Middleton class-A noise, MMSE, MC-CDMA, multipath fading, orthogonal poly-phase codes, power-line communications.

\section{INTRODUCTION}

The fact that broadband power-line communication (BB-PLC) creates one consolidated network, instead of two, makes it more appealing than its competitors since devices connected to this network (computers, screens, CCTVs etc.) are always plugged into the electrical sockets regardless [1]. Power-line channels, however, are a very harsh and noisy transmission medium suffering from frequency selectivity, time-varying topology issues, electromagnetic interference and noise. Many studies have concluded that noise is the most crucial element influencing communication signals over PLC channels and is, in general, classified into background noise (BN) and impulsive noise (IN) [2]-[4]. In particular, IN can have very high amplitudes with durations frequently exceeding the signal symbol length which can dramatically affect high speed communications [5]. In order to reduce the adverse effect of this noise, blanking, clipping, or a combination of both (hybrid), is usually applied at the front-end of the orthogonal frequencydivision multiple access (OFDM) receiver to zero or/and clip the incoming signal when it exceeds a certain threshold value [6], [7].

Unlike other studies, which are OFDM-based only, in this paper we study the application of multi-carrier code-division multiple access (MC-CDMA) with orthogonal poly-phase (OPP) sequences combined with blanking, clipping and adaptive hybrid over multipath BB-PLC channels. The rationale for selecting OPP codes, which are the non-binary extension of the binary WH sequences, includes: firstly, their robustness against timing misalignment which can significantly degrade the performance of the binary WH codes. Secondly, OPP codes have similar crosscorrelation properties as the binary $\mathrm{WH}$ and, unlike the latter,
OPP codes are not limited by the sequence length hence can accommodate much more users/data. Thirdly, but most importantly, such codes tend to have less peak-to-average power ratio (PAPR) properties as the constellation size of these codes is increased [8]. The utmost importance of this resides in the fact that minimizing the PAPR of the transmitted signal will allow more efficient blanking and/or clipping of IN at the receiver and hence improve the overall performance [9]. It should also be mentioned that a minimum-mean square error (MMSE) equalizer is employed at the receiver to compensate for the inter-symbol interference caused by the frequency-selective channel without enhancing the noise power.

The contribution of this paper is threefold. First the PAPR performance of the OPP-MC-CDMA approach is studied for various constellation sizes of OPP codes under full-loading and half-loading system scenarios to establish the critical relationship between the system loading, OPP code constellation size and PAPR of the transmitted signal. This is important as it was shown [9] that signals with lower PAPR are more resilient to bursty noise. The second contribution resides in evaluating the signal-to-noise ratio (SNR) value at the output of the most three popular nonlinear preprocessors for reducing the impact of bursty noise in multipath fading. Finally, the problem of blanking and clipping thresholds optimization is addressed in different noise environments and the corresponding maximum achievable output SNR and minimum symbol error rate (SER) performances are evaluated. For the purpose of comparison and completeness, OFDM-based schemes are also included throughout our investigations. The results indicate that OPP-MC-CDMA with blanking and clipping can offer up to $5.25 \mathrm{~dB}$ and $2.25 \mathrm{~dB}$ improvement in the output SNR over the OFDM-based systems, respectively. It is also shown that the proposed system is always able to considerably enhance performance even when the constellation size of OPP codes is relatively small.

The rest of this paper is organized as follows. In Section II the system model is described. Section III discusses the PAPR performance of the proposed system for various OPP codes phases. Section IV is dedicated to study the output SNR. In Section $\mathrm{V}$ the maximum achievable output SNR and the minimum SER performances that correspond to the optimal blanking and clipping thresholds are examined for various noise scenarios. The impact of Middleton class-A impulsive index on the over all performance of the optimized system is outlined in Section VI. Finally, Section VII gives a summary and some concluding remarks.

\section{System Model}

The system model under consideration is illustrated in Fig. 1. First, the 16-QAM symbol $s_{m}$ of each user is spread using the user-specific code $\mathbf{c}_{m}=\left[c_{m}^{(0)}, c_{m}^{(1)}, \ldots, c_{m}^{(N-1)}\right]$, where $m=$ 


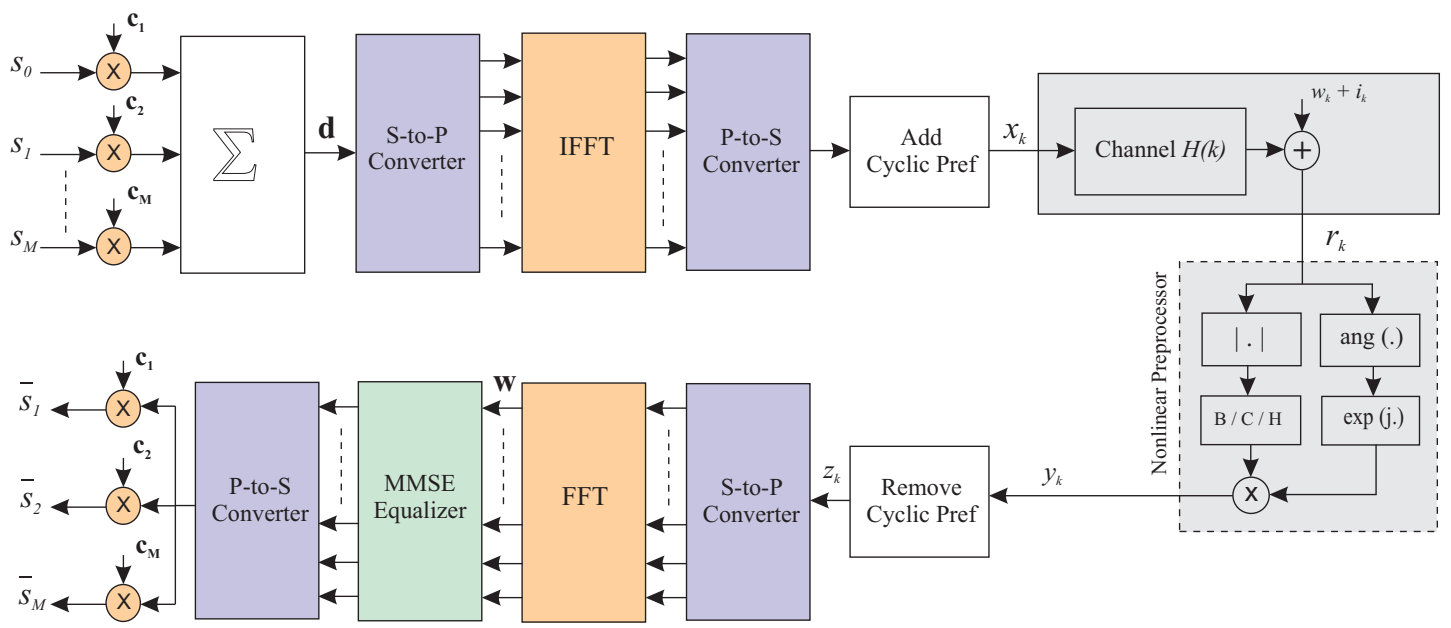

Figure 1: Block diagram of the OPP-MC-CDMA system with a nonlinear preprocessing device at the receiver.

$[1,2, \ldots, M], M$ is the total number of users and $N$ denotes the code length. It should be stated here that, throughout this work unless explicitly stated otherwise, we use a fully loaded system (i.e. $M=64$ users). After that, the spread signals are multiplexed to produce $\mathbf{d}=\left[d_{0}, d_{1}, \ldots, d_{N-1}\right]$ which is then passed through a serial-to-parallel (S-to-P) converter. The S-to-P output is fed to an inverse fast Fourier transform (IFFT), the size of which is equal to the code length $(N)$, then applied to a parallel-to-serial (P-to-S) converter and a cyclic prefix (CP) is added before transmission. The transmitted signal for one MC-CDMA block is expressed mathematically as

$$
x(t)=\sum_{i=0}^{N-1} \sum_{m=0}^{M-1} s_{m} c_{m}^{(i)} e^{\left(j \frac{2 \pi i t}{T_{s}}\right)}
$$

where $T_{s}$ is the MC-CDMA symbol duration. Using this definition in (1), the PAPR of the MC-CDMA transmitted signal is given by

$$
\text { PAPR }=\frac{\max \left[|x(t)|^{2}\right]}{\mathrm{E}\left[|x(t)|^{2}\right]}
$$

where $E[$.$] is the expectation operator. After the signal is passed$ through the multipath PLC channel, the received signal can be written as

$$
r_{k}=x_{k} * h_{k}+n_{k}, \quad k=0,1, \ldots, N-1
$$

where $x_{k}=x\left(k T_{s} / N L\right),{ }^{\prime} *^{\prime}$ denotes convolution operation, $h_{k}$ is the impulse response of multipath fading channel and $n_{k}$ is the total noise component. It should be pointed out that perfect channel estimation is assumed and that orthogonality is maintained by the cyclic prefix. In order to emulate both $\mathrm{BN}$ and IN, Middleton class-A noise model, [10], [11], is adopted here with the following probability density function (PDF)

$$
p(z)=\sum_{m=0}^{\infty} \frac{e^{-A} A^{m}}{m !} \cdot \frac{1}{\sqrt{2 \pi \sigma_{m}^{2}}} \exp \left(-\frac{|z|^{2}}{2 \sigma_{m}^{2}}\right)
$$

where

$$
\sigma_{m}^{2}=\sigma_{u}^{2}\left(\frac{\frac{m}{A}+\Gamma}{1+\Gamma}\right), \sigma_{u}^{2}=\sigma_{G}^{2}+\sigma_{I}^{2} \text { and } \Gamma=\frac{\sigma_{G}^{2}}{\sigma_{I}^{2}}
$$

while $\sigma_{m}^{2}$ denotes the variance of the $m^{\text {th }}$ considered IN source, $\sigma_{u}^{2}$ is the total noise power, $\sigma_{G}^{2}$ is the Gaussian noise power, $\sigma_{I}^{2}$ is the impulsive (non-Gaussian) noise power, $A=E\{m\}=$ $\sum_{m=0}^{\infty}\left(m e^{-A} A^{m} / m !\right)$ representing the average number of impulsive sources simultaneously active and is referred to as impulsive index and $\Gamma$ denotes the ratio between the average power of the Gaussian component and the impulsive component. It is clear that the three parameters $A, \Gamma$ and $\sigma_{u}^{2}$ specify the statistical characteristic of this model and that when $A$ is large IN will become continuous and, therefore, Middleton class-A noise becomes more likely as Gaussian noise; while conversely, low values of $A$ means rare and highly structured IN. To visualize this, numerical results, obtained from (4), are illustrated in Fig. 2 for various values of $A$ when $\Gamma=0.01$ along with the Gaussian PDF. It is apparent that when $A$ is large $(A=10)$ the distribution of Middleton class-A noise is very similar to Gaussian distribution but becomes more impulsive as $A$ is reduced.

In order to diminish the deleterious impact of IN, the received signal is passed through one of the following nonlinear preprocessors

- Blanking

$$
y_{k}=\left\{\begin{array}{ll}
r_{k}, & \left|r_{k}\right| \leq T_{b} \\
0, & \left|r_{k}\right|>T_{b}
\end{array} \quad k=0,1, \ldots, L N-1\right.
$$

where $T_{b}$ is the blanking threshold.

- Clipping

$$
y_{k}=\left\{\begin{array}{ll}
r_{k}, & \left|r_{k}\right| \leq T_{c} \\
T_{c} e^{j \arg \left(r_{k}\right)}, & \left|r_{k}\right|>T_{c}
\end{array} \quad k=0,1, \ldots, L N-1\right.
$$

where $T_{c}$ is the clipping threshold.

- Adaptive Hybrid

$$
y_{k}= \begin{cases}r_{k}, & \left|r_{k}\right| \leq T \\ \alpha T e^{j \arg \left(r_{k}\right)}, & T<\left|r_{k}\right| \leq \alpha T \\ 0, & \left|r_{k}\right|>\alpha T\end{cases}
$$

while $r_{k}$ and $y_{k}$ are the input and output of the nonlinear devices, respectively; $T$ and $\alpha$ are the threshold and scaling factor $(\alpha>1)$ of the adaptive hybrid scheme, respectively, with the latter defining the ratio between the blanking and clipping thresholds. Above all, the adaptive hybrid scheme was found to establish the lower bound performance of the nonlinear preprocessing-based method since it 


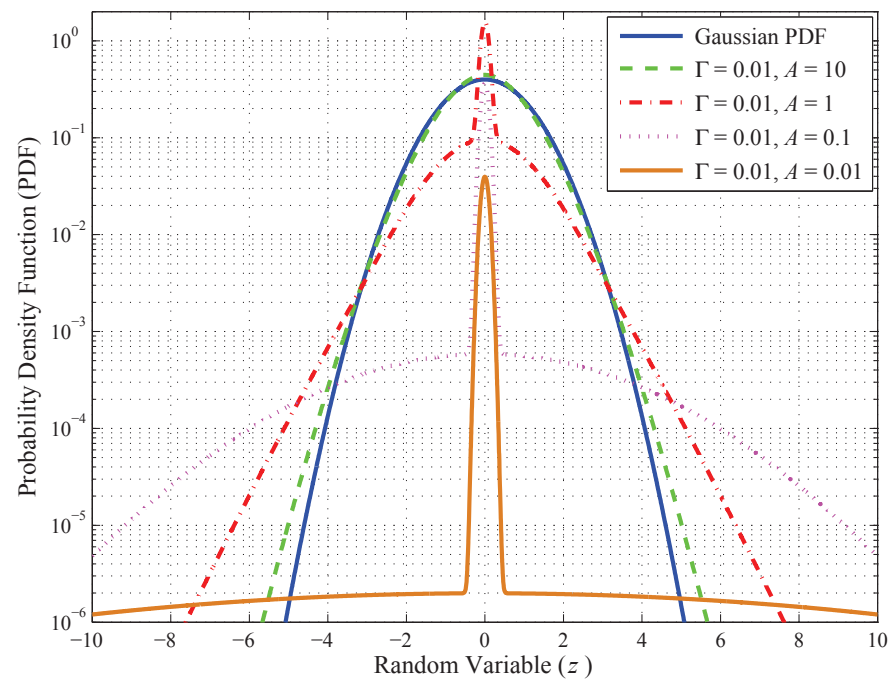

Figure 2: PDFs of Middleton class-A noise for different values of A when $\Gamma=0.01$.

optimizes not only the threshold but also the scaling factor [12].

After the nonlinear device, the next stage is the $\mathrm{CP}$ removal after which the resultant signal $\left(z_{k}\right)$ is fed to the S-to-P converter and then to an $M$-point fast Fourier transform (FFT) to produce $\mathbf{W}=\left[W_{0}, W_{1}, \ldots, W_{N-1}\right]$. In order to compensate the channel distortion, equalization is performed using the MMSE equalizer as

$$
W(k)=\frac{H^{*}(k)}{H(k) H^{*}(k)+\mathrm{SNR}_{\text {out }}^{-1}}
$$

where $\mathrm{SNR}_{\text {out }}$ represents the SNR at the output of the nonlinear preprocessor. After that, the output of the MMSE equalizer is passed through a P-to-S converter which is then multiplied by the spreading codes to produce estimates for the data symbols of the different users $\left(\bar{s}_{m}\right)$. Finally, 16-QAM demodulation takes place and SER is calculated.

\section{PAPR RELATIONSHIP TO OPP CONSTELLATIONS}

In this section we investigate the impact of the number of OPP codes phases $(\phi)$ on the PAPR performance. Before proceeding with this, however, the complementary cumulative distribution function (CCDF) of the PAPR should be introduced. It is defined as the probability that the PAPR of a data block exceeds a given threshold $\left(\mathrm{PAPR}_{o}\right)$ and is expressed as $\mathrm{CCDF}=1-P(\mathrm{PAPR} \leq$ $\mathrm{PAPR}_{o}$ ). Fig. 3 depicts the PAPR performance of the OPPMC-CDMA signal against $\phi$ for two loading scenarios: halfloading $(M=32)$ and full-loading $(M=64)$ when $\mathrm{CCDF}=$ $10^{-1}, 10^{-2}$ and $10^{-3}$. It is clear that the PAPR value is reduced with increasing the number of OPP codes phases. Comparing the two loading scenarios, it is obvious that full-loading has always lower PAPR with respect to half-loading at the same CCDF and $\phi$ values. It is also apparent that the amount of PAPR reduction is more significant in full-loading than that with halfloading. For example, in the former case at $\mathrm{CCDF}=10^{-3}$, a PAPR reduction of about $6 \mathrm{~dB}$ can be achieved when $\phi=64$ relative to the system with $\phi=2$ whereas only about $4.5 \mathrm{~dB}$ is attained from the half-loading scenario at the same CCDF value. This enhancement can be intuitively justified by the increase in the phase randomization across the sub-carriers which is related

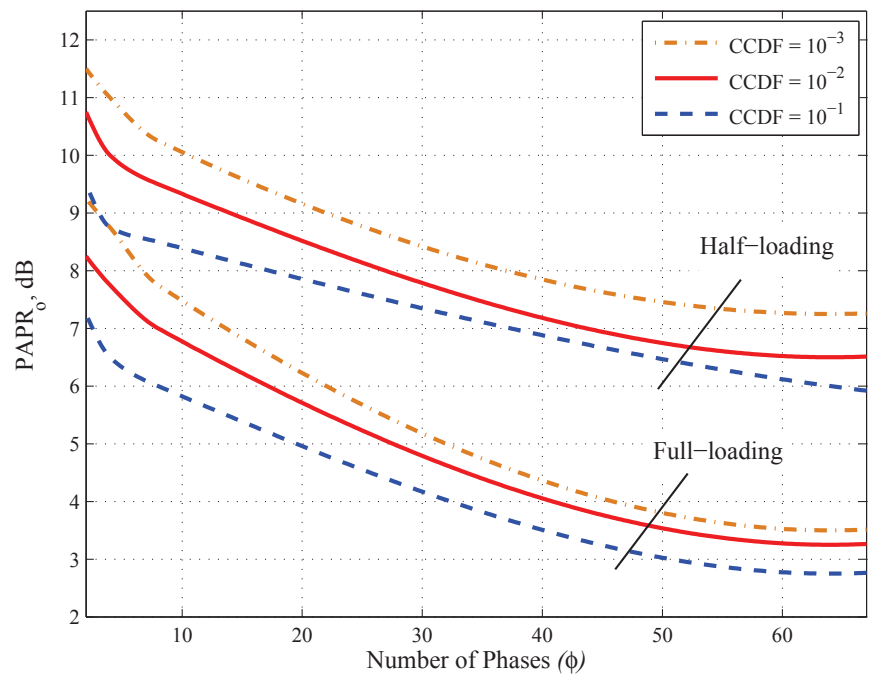

Figure 3: PAPR of OPP-MC-CDMA system versus the constellation size of OPP codes for various CCDF values with full-loading and half-loading.

to the minimum-distance decoding of the sequences [13]. This, eventually, leads to more averaging of the transmitted MC-CDMA signal and, consequently, improves the overall PAPR performance. Recalling the OFDM-based results found in [9], minimizing the signals' peaks implies that IN will become more easily identifiable at the receiver and, subsequently, will allow more accurate and efficient noise cancellation.

Although in this section we looked into both half-loading and full-loading scenarios, in the rest of this paper, without loss of generality, the focus will be primarily on the latter not only because it relatively has better PAPR properties but also because full loading is a more common configuration in practice.

\section{PReProcessor Threshold IMPACT ON OUtPUt SNR}

In this section we assess the performance of OPP-MC-CDMA with different constellation sizes in terms of SNR at the output of the nonlinear preprocessors which is determined as

$$
\mathrm{SNR}_{\text {out }}=\frac{2 K_{o}^{2}}{E_{\text {out }}-2 K_{o}^{2}}
$$

where $K_{o}$ is determined as $K_{o}=(1 / 2) E\left[\left|y_{k} x_{k}^{*}\right|\right]$ and $E_{\text {out }}=$ $E\left[\left|y_{k}\right|^{2}\right]$. Our investigations from this section onward, unless stated otherwise, are based on: $M=64$ users, transmitted signal is normalized as $\sigma_{x}^{2}=(1 / 2) E\left[\left|x_{k}\right|^{2}\right]=1, \sigma_{u}=0.05, A=0.0025$ and $\Gamma=0.001$ which means that IN is 1000 times, or 30 $\mathrm{dB}$, greater than the BN level. Fig. 4 shows the output SNR of the proposed system versus the blanking/clipping threshold for the three nonlinear preprocessing schemes with different OPP codes phases $\{\phi=2,4,8,64\}$ in a multipath fading channel. In addition, the performance of OFDM-based schemes is also included with and without multipath fading. It should be mentioned that the analytical results of the OFDM system W/O multipath for the blanking and clipping techniques are found using (10) with $K_{O}$ and $E_{\text {out }}$ expressions derived in [7]; whereas, for the adaptive hybrid, these parameters are presented in [12] for the two component mixture-Gaussian noise model and are rewritten here for the general Middleton class-A noise model as in (11) and (12), respectively. 


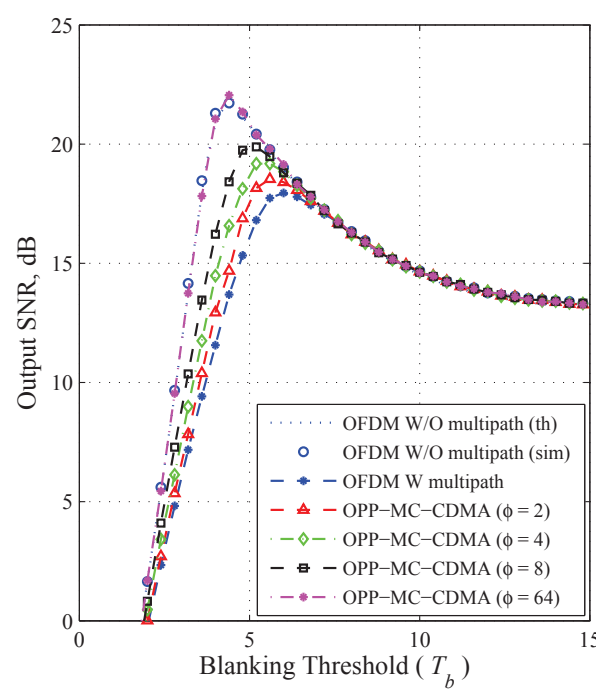

(a) Blanking

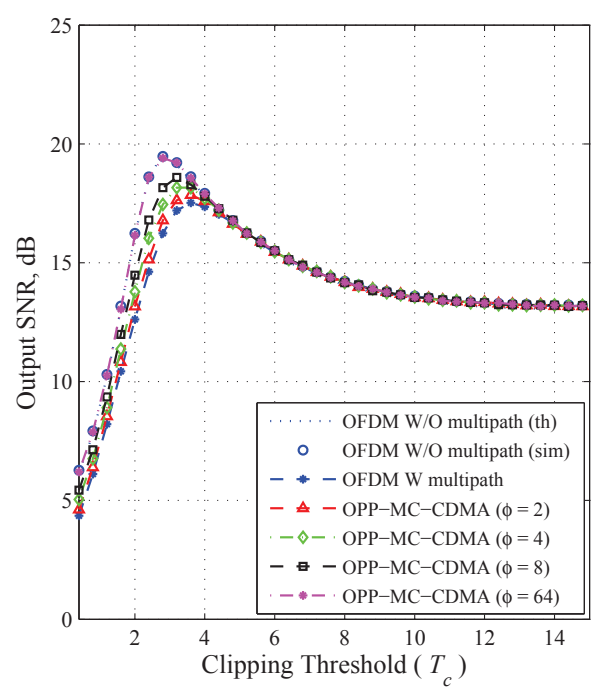

(b) Clipping

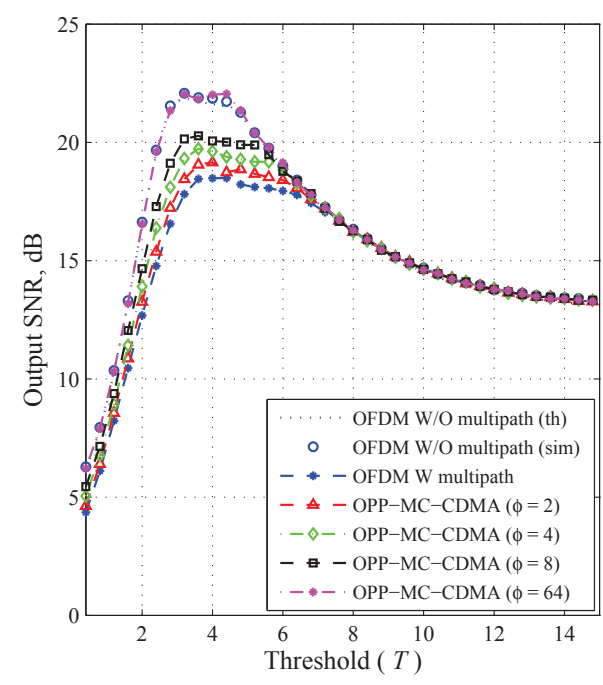

(c) Adaptive Hybrid

Figure 4: Output SNR as a function of the blanking/clipping threshold for the OPP-MC-CDMA system with various values of $\phi$ and OFDM system, with and without multipath in the presence of Middleton class-A noise. Noise parameters are $\sigma_{u}=0.05, \Gamma=0.001$ and $A=0.0025$.

$$
\begin{gathered}
K_{\text {o }}=1-\sum_{m=0}^{\infty} p_{m}\left[e^{-\frac{T^{2}}{2\left(1+\sigma_{m}^{2}\right)}}+\frac{T^{2}}{2\left(1+\sigma_{m}^{2}\right)} e^{\left.-\frac{\alpha^{2} T^{2}}{2\left(1+\sigma_{m}^{2}\right)}\right]}\right]-\sum_{m=0}^{\infty} p_{m} \sqrt{\frac{\pi}{2}} \frac{T}{\sqrt{1+\sigma_{m}^{2}}}\left[Q\left(\frac{T}{\sqrt{1+\sigma_{m}^{2}}}\right)-Q\left(\frac{\alpha T}{\sqrt{1+\sigma_{m}^{2}}}\right)\right] \\
E_{\text {out }}=2+2 \sum_{m=0}^{\infty} p_{m}\left(\sigma_{m}^{2}-\left(1+\sigma_{m}^{2}\right) e^{-\frac{T^{2}}{2\left(1+\sigma_{m}^{2}\right)}}-\frac{T^{2}}{2} e^{-\frac{\alpha^{2} T^{2}}{2\left(1+\sigma_{m}^{2}\right)}}\right)
\end{gathered}
$$

As anticipated, it is obvious that, in a multipath channel and irrespective of the nonlinear device utilized, the performance of the OPP-MC-CDMA system always outperforms the OFDM approach even with a small number of phases (e.g. $\phi=2)$ and this improvement becomes higher as the code constellation size is increased. Clearly, the proposed system with $\phi=64$ can offer output SNR gains of up to $4 \mathrm{~dB}, 2 \mathrm{~dB}$ and $4 \mathrm{~dB}$ relative to the OFDM-based one when blanking, clipping and adaptive hybrid are employed, respectively. Interestingly enough, however, it is evident that when the constellation size of the OPP codes is sufficiently large $(\phi=64)$, OPP-MC-CDMA can always achieve exact performance as OFDM W/O multipath. Notably, in both OFDM and OPP-MC-CDMA approaches, the adaptive hybrid scheme serves as an upper bound for the performance compared to blanking and clipping cases. Another general trend can be noticed for the three aforementioned nonlinear devices that is when the blanking and/or clipping threshold is very large (typical receiver), the output SNR approaches $13 \mathrm{~dB}$ in which scenario it can be expressed mathematically as

$$
\operatorname{SNR}_{\text {out }}\left(T_{b, c, a h} \rightarrow \infty\right)=\left(\sigma_{u}^{2} \sum_{m=0}^{\infty} p_{m}\left(\frac{\frac{m}{A}+\Gamma}{1+\Gamma}\right)\right)^{-1}
$$

As a final remark on the results in this section, it is interesting to observe that for every phase value there is always an optimal blanking/clipping threshold that maximizes output SNR performance.

\section{Performance Optimization}

In this section extensive computer simulations are conducted to find the maximum achievable output SNR and minimum SER performance that correspond to the optimal blanking/clipping threshold for the OPP-MC-CDMA and OFDM systems under various noise scenarios. From this point onward, only blanking and clipping are considered, not only because adaptive hybrid was shown to offer insignificant improvement compared to the other two nonlinear devices, but also because it is more complex to achieve optimal performance as it requires optimizing two parameters $(T$ and $\alpha)$, instead of one. We examine here the optimized system performance when $\sigma_{u}^{2}$ is varied while fixing $\Gamma$. With this mind, the maximum achievable output SNR is plotted versus $10 \log _{10}\left(\sigma_{u}^{2}\right)$ as shown in Figs. 5(a) and (b) for the systems under consideration with blanking and clipping, respectively, when $A=0.0025$ and $\Gamma=0.001$. The analytical results of OFDM W/O multipath are found from (10) by satisfying

$$
\min _{T_{b, c}}\left\{\frac{E_{\text {out }}}{K_{o}^{2}}\right\}
$$

Comparing Figs. 5(a) and (b), a number of common observations can be seen. Firstly, the typical receiver always has the worst performance followed by the OFDM system with multipath. Secondly, the MC-OPP approach always outperforms the OFDMbased schemes even with a small value of $\phi$ and this enhancement becomes higher as $\phi$ is increased. The third common trend in these figures, and the most interesting, is that the proposed system with $\phi=64$ approaches the performance of OFDM W/O multipath 


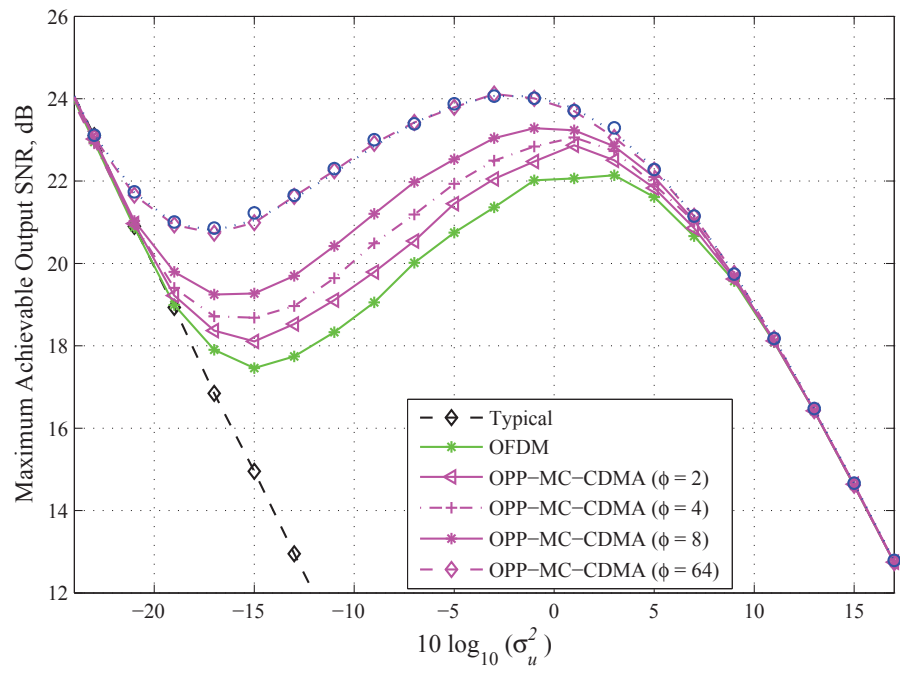

(a) Blanking

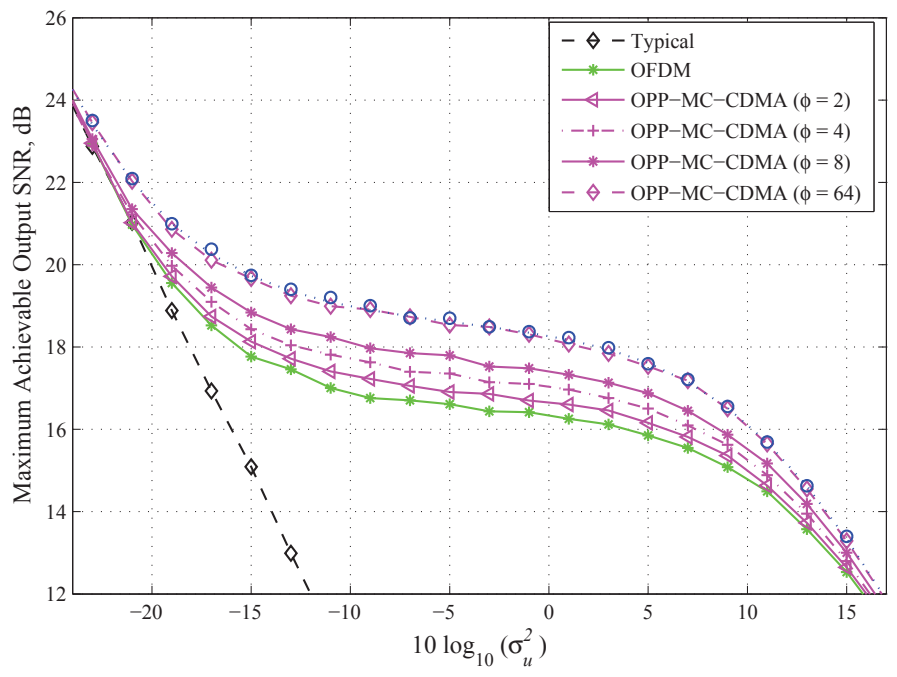

(b) Clipping

Figure 5: Maximum achievable output SNR corresponding the optimal blanking/clipping threshold versus $10 \log _{10}\left(\sigma_{u}^{2}\right)$ for the OFDM and MC-CDMA systems in a multipath channel for various codes phases with blanking and clipping. Noise parameters are $A=0.0025$ and $\Gamma=0.001$. OFDM with no multipath: (dotted line $\rightarrow$ analytical), ( $\circ \rightarrow$ simulation).

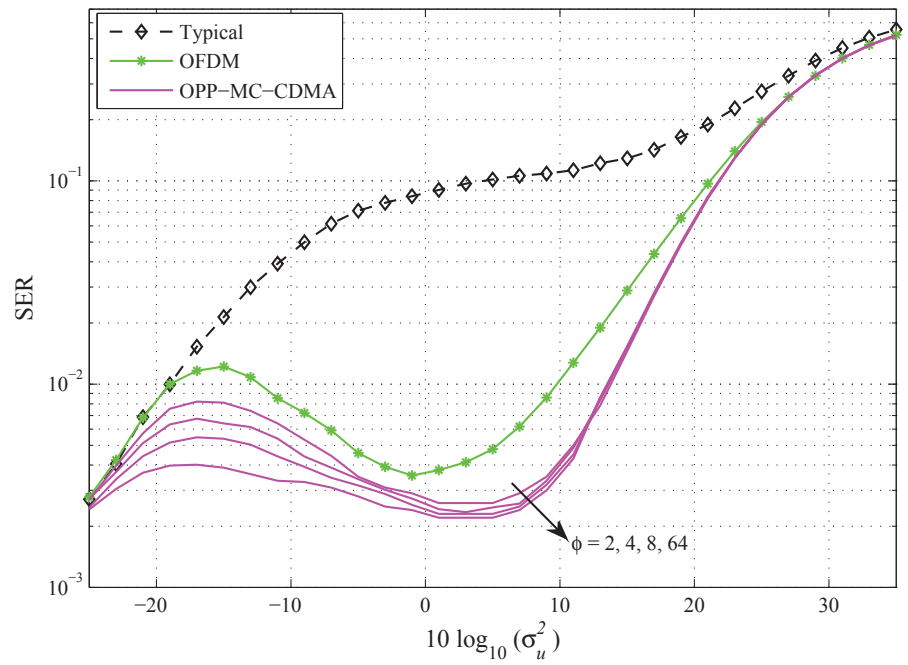

(a) Blanking

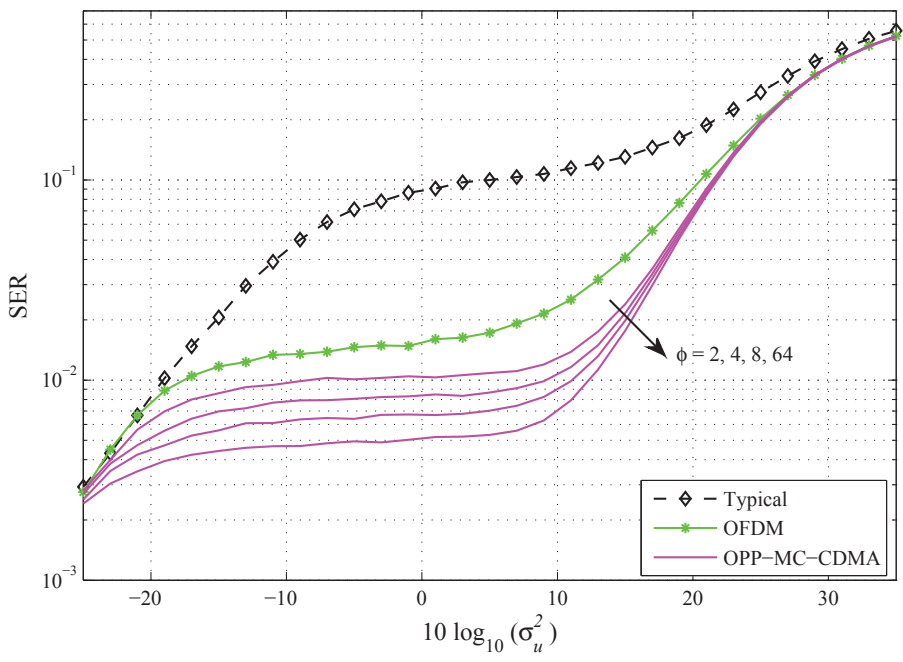

(b) Clipping

Figure 6: Minimum SER performance corresponding to the maximum achievable output SNR versus $10 \log _{10}\left(\sigma_{u}^{2}\right)$ for the OFDM and MC-CDMA systems in a multipath channel for various codes phases with blanking and clipping. Noise parameters are $A=0.0025$ and $\Gamma=0.001$.

over the entire noise spectrum. In general, it is also noticeable that blanking always yields better performance than clipping under same noise and modulation features. Moreover, when $\sigma_{u}^{2}$ is very low, around $25 \mathrm{~dB}$ below transmitted signal level, the performance is very good for both blanking and clipping which is intuitive. At the other extreme, however, when $\sigma_{u}^{2}$ is very high, around $10 \mathrm{~dB}$ above the signal level, performance deteriorates dramatically. This can be justified by the fact that in this noise region, the system becomes Gaussian limited making the nonlinear preprocessing-based schemes inefficient. In the intermediate $\sigma_{u}^{2}$ region $\left\{-25 \mathrm{~dB} \lesssim \sigma_{u}^{2} \lesssim 10 \mathrm{~dB}\right\}$, the output SNRs of the blankingbased systems degrade as $\sigma_{u}^{2}$ increases until it reaches about $-15 \mathrm{~dB}$ after which performance begins to improve reaching its peak at around $\sigma_{u}^{2}=0 \mathrm{~dB}$ with an output SNR of $24 \mathrm{~dB}$ before it declines rapidly again. On the other hand, for the clipping case, the output SNR consistently decreases as $\sigma_{u}^{2}$ increases over the entire intermediate region of $\sigma_{u}^{2}$.

For more quantitative characterization of the proposed system, the SER performance in correspondence to the SNRs curves shown in Fig. 5 is found and presented in Fig. 6 from which similar observations can be seen. Notably, blanking has generally lower SER performance but when for $\sigma_{u}^{2}$ is extremely high, SER reaches 1 for all considered systems indicating that the nonlinear devices are no longer efficient and, as a consequence, more sophisticated techniques should be utilized instead.

\section{IMPULSIVE INDEX IMPACT ON THE OPTIMIZED SySTEMS}

In this section we investigate the impact of the impulsive index $(A)$ on the performance of the proposed system. To do this, we set the noise power as $\sigma_{u}^{2}=0.05$ and plot the output SNR of OPP-MC-CDMA with blanking and clipping versus $\phi$ for $A=0.001,0.003,0.005,0.01$, and 0.05 as shown in Fig. 7 . It is 


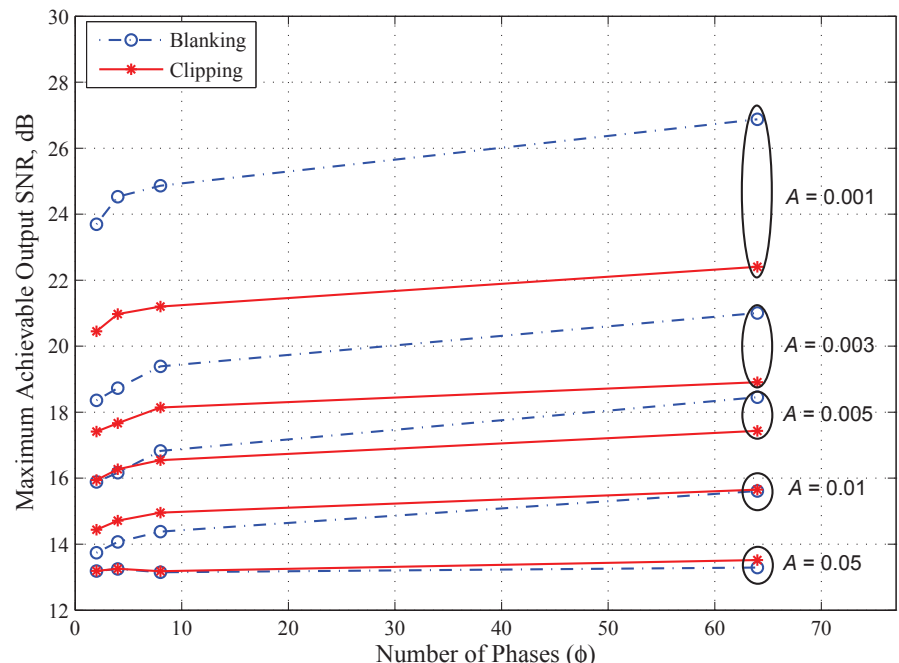

Figure 7: Maximum achievable output SNR versus the number of OPP codes phases with different values of $A$ for both blanking and clipping in a multipath channel. Noise parameters are $\sigma_{u}=0.05$ and $\Gamma=0.001$.

clearly visible that the output SNR is inversely proportional to $A$ but, as expected, directly proportional to $\phi$. For instance, when $A=0.001$, gains of up to $3 \mathrm{~dB}$ and $2 \mathrm{~dB}$ are attained when $\phi=64$ relative to the case when $\phi=2$ for the blanking and clipping, respectively; whereas when $A=0.005$ these gains are reduced to around $2.5 \mathrm{~dB}$ and $1.5 \mathrm{~dB}$ for the same features. It is also obvious that the OPP blanking-based scheme performs better than the clipping one when $A$ is low (e.g. $A=0.001,0.003$ and 0.005 ) whereas clipping offers slightly better performance when $A$ is relatively high (e.g. $A=0.01$ and 0.05 ). Furthermore, it is interesting to note that in a heavily-disturbed IN environment $(A=0.05)$ the output SNR becomes almost independent of $\phi$ for both nonlinear preprocessing-based systems. However, it was presented in [2] that such a high IN probability of occurrence is very rare in practice.

For better visualization of the achievable gain over the OFDM-based scheme, we plot in Fig. 8 the relative gain ( $\left.G_{\text {OPP-MC-CDMA }}\right)$, which is basically the ratio between the output SNRs of the OPP-MC-CDMA and the OFDM-based systems and is given by

$$
G_{\mathrm{OPP}-\mathrm{MC}-\mathrm{CDMA}}=10 \log _{10}\left(\frac{\mathrm{SNR} \text { OPP }-\mathrm{MC}-\mathrm{CMDA}}{\mathrm{SNR}_{\mathrm{OFDM}}}\right)
$$

This figure depicts $G_{\mathrm{OPP}-\mathrm{MC}-\mathrm{CDMA}}$ versus $\phi$ for several values of $A$ from which it is evident that when $A=0.001$ gains of up to $5.25 \mathrm{~dB}$ and $2.25 \mathrm{~dB}$ can be achieved when $\phi=64$ for the blanking and clipping scenarios, respectively. Notably, in a heavilydisturbed IN environment, $G_{\mathrm{OPP}-\mathrm{MC}-\mathrm{CDMA}}$ becomes negligible for clipping and zero for blanking which implies that in such IN scenarios OFDM system should be implemented instead since it is less complex.

\section{CONCLUSION}

In this paper we explored the implementation of OPP-based MC-CDMA over frequency-selective power-line channels contaminated with Middleton class-A noise. Three burst noise reduction techniques were considered, namely, blanking, clipping and adaptive hybrid. The problem of blanking and clipping threshold optimization was also studied. Compared to the OFDM-based scheme, it was found that the proposed system is more effective

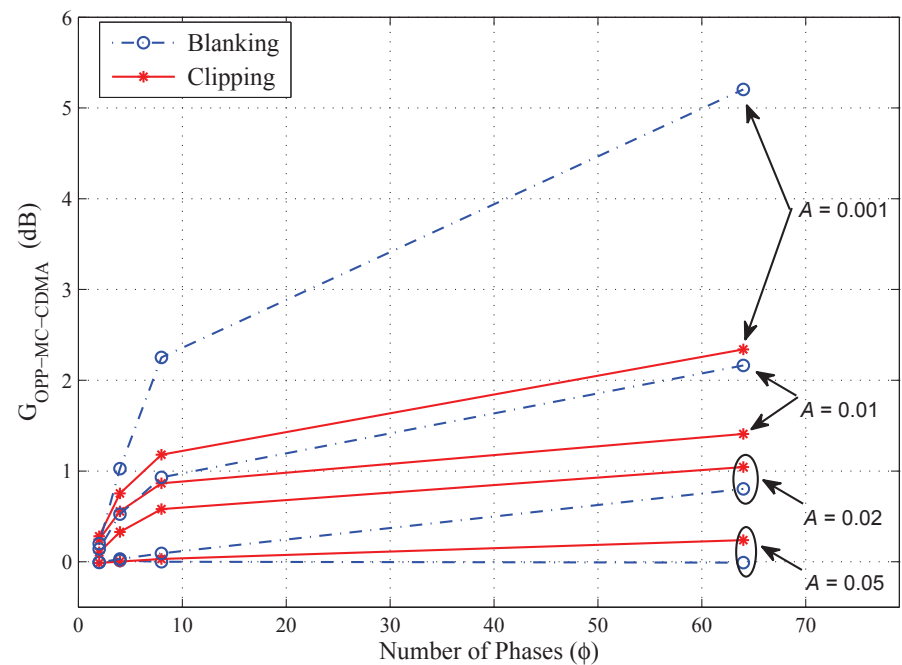

Figure 8: The gain relative to the OFDM-based system against $\phi$ with several values of $A$ for both blanking and clipping in a multipath channel. Noise parameters are $\sigma_{u}=0.05$ and $\Gamma=0.001$.

in tackling the PLC channel impairments providing output SNR gains of more than $5 \mathrm{~dB}$ and $2 \mathrm{~dB}$ for the blanking- and clippingbased systems, respectively. Moreover, it was demonstrated that the performance of the OPP-MC-CDMA approach improves as we increase the constellation size of the OPP codes.

\section{REFERENCES}

[1] S. Galli, A. Scaglione, and K. Dostert, "Broadband is power: internet access through the power line network," IEEE Commun. Mag., vol. 41, no. 5, pp. 82-83, May 2003.

[2] M. Zimmermann and K. Dostert, "Analysis and modeling of impulsive noise in broad-band powerline communications," IEEE Trans. Electromagn. Compat. vol. 44, pp. 249-258, Feb. 2002.

[3] M. H. L. Chan and R. Donaldson, "Amplitude, width, and interarrival distributions for noise impulses on intrabuilding power line communication networks," IEEE Trans. Electromagn. Compat., vol. 31, no. 3, pp. 320-323, Aug. 1989.

[4] H. Meng, Y. L. Guan, and S. Chen, "Modeling and analysis of noise effects on broadband power-line communications," IEEE Trans. Power Del., vol. 20, no. 2, pp. 630-637, Apr. 2005.

[5] M. Gotz, M. Rapp, and K. Dostert, "Power line channel characteristics and their effect on communication system design," IEEE Commun. Mag., vol. 42, no. 4, pp. 78-86, Apr. 2004.

[6] K. Rabie and E. Alsusa, "Quantized peak-based impulsive noise blanking in power-line communications," IEEE Trans. Power Del., vol. 29, no. 4, pp. 1630-1638, Aug. 2014.

[7] S. V. Zhidkov, "Analysis and comparison of several simple impulsive noise mitigation schemes for OFDM receivers," IEEE Trans. Commun., vol. 56, no. 1, pp. 5-9, Jan. 2008.

[8] K. Rabie and E. Alsusa, "MC-CDMA transmission with blanking nonlinearity for impulsive noise power-line communication channels," in Proc. IEEE Veh. Technol. Conf. (VTC), May 2015, to appear.

[9] - "Preprocessing based impulsive noise reduction for power-line communications," IEEE Trans. Power Del., vol. 29, no. 4, pp. 1648-1658, Aug. 2014.

[10] D. Middleton, "Statistical-physical models of urban radio-noise environments - part I: Foundations," IEEE Trans. Electromagn. Compat., vol. EMC-14, no. 2, pp. 38-56, May 1972.

[11] _ "Statistical-physical models of electromagnetic interference," IEEE Trans. Electromagn. Compat., vol. EMC-19, pp. 106-127, Aug. 1977.

[12] K. Rabie and E. Alsusa, "Threshold and scaling factor optimization for enhancing impulsive noise cancellation in PLC systems," in Proc. IEEE Global Commun. Conf. (GLOBECOM), Dec. 2014, to appear.

[13] V. Tarokh and H. Jafarkhani, "On the computation and reduction of the peak-to-average power ratio in multicarrier communications," IEEE Trans. Commun., vol. 48, no. 1, pp. 37-44, Jan. 2000. 